\title{
Ultrasensitive Ultrafast Vibrational Spectroscopy Employing the Near Field of Gold Nanoantennas
}

\author{
O. Selig, R. Siffels, and Y.L. A. Rezus* \\ FOM Institute AMOLF, Science Park 104, 1098 XG Amsterdam, Netherlands
}

(Received 17 September 2014; published 12 June 2015)

\begin{abstract}
We introduce a novel method to perform nonlinear vibrational spectroscopy on nanoscale volumes. Our technique uses the intense near field of infrared nanoantennas to amplify the nonlinear vibrational signals of molecules located in the vicinity of the antenna surface. We demonstrate the capabilities of the method by performing infrared pump-probe spectroscopy and two-dimensional infrared spectroscopy on $5 \mathrm{~nm}$ layers of polymethylmetacrylate. In these experiments we observe enhancement factors of the nonlinear signals of more than 4 orders of magnitude. We discuss the mechanism underlying the amplification process as well as strategies for further increasing the sensitivity of the technique.
\end{abstract}

DOI: 10.1103/PhysRevLett.114.233004

PACS numbers: 33.20.Ea, 78.47.J-, 78.67.Qa

Vibrational spectroscopy is a powerful, label-free technique that provides detailed information about molecular structures. The method uses vibrational marker modes to identify the chemical groups present inside a molecule as well as to probe their chemical nanoenvironments. In recent years vibrational spectroscopy has experienced a revival with the advent of novel, nonlinear vibrational spectroscopies, in particular, infrared pump-probe spectroscopy and two-dimensional infrared (2DIR) spectroscopy [1-5]. In these techniques the sample is interrogated by a number of time-delayed femtosecond infrared pulses, allowing one to study not only the structures of molecules but also their dynamics [6-9]. While these two nonlinear methods are mostly applied to macroscopic samples, they could in principle provide valuable information about nanoscale systems consisting of molecular monolayers, such as the active areas of biosensors, supported lipid bilayers, and responsive surfaces $[10,11]$. Unfortunately, because of the low absorption cross section of molecular vibrations, the only molecular monolayers that are amenable to study using these techniques are those composed of extremely strong infrared absorbers: in particular, to date such measurements have only been reported for metal-carbonyl compounds $[12,13]$. Obviously, a method allowing for the application of these nonlinear spectroscopies to other, less absorptive, molecular vibrations at the (sub)monolayer level would be highly beneficial.

An attractive option for amplifying the interaction of molecular vibrations with infrared radiation is the use of optical nanoantennas. These metallic nanostructures have recently attracted considerable interest due to their ability to funnel light to subwavelength volumes and thereby strongly enhance the local electromagnetic field $[14,15]$. Particularly strong field enhancements can be achieved with the help of resonant nanostructures, such as rodshaped nanoantennas, which exhibit a dipolar resonance that can be tuned all the way from the visible spectral region to the far infrared, simply by varying the length of the nanoantenna. In the past it has been demonstrated that infrared-resonant nanoantennas can strongly amplify the linear vibrational spectroscopic signals of molecules located in their near fields [16-20]. In addition, nonlinear frequency-mixing processes such as second-harmonic generation [21], third-harmonic generation [22], and four-wave mixing $[23,24]$ have been demonstrated to occur in the near fields of nanoantennas. An application that has not been explored to date is the use of infrared nanoantennas as a means to perform highly efficient nonlinear spectroscopy. Recently, it was demonstrated that nonlinear vibrational signals (2DIR) can be enhanced using colloidal gold nanoparticles [25], but the possibilities provided by nanoantenna structures designed to be resonant with a specific vibrational mode have not yet been exploited.

Here we report on a proof-of-principle experiment in which we use the enhanced near field of nanoantenna arrays to perform femtosecond vibrational pump-probe spectroscopy on the carbonyl vibration of ultrathin layers of polymethylmetacrylate (PMMA) deposited on top of the arrays. The enhanced near field of the nanoantennas decays rapidly with distance, and, consequently, we only probe those regions of the PMMA film located directly on top of the nanoantennas. We demonstrate that with this method the nonlinear spectroscopic signals are amplified by more than 4 orders of magnitude.

In our experiments we use gold nanoantenna arrays that are fabricated on $\mathrm{CaF}_{2}$ substrates using standard $e$-beam lithography. The antennas are arranged in $800 \mu \mathrm{m} \times$ $800 \mu \mathrm{m}$ randomized arrays at a density of $0.17 \mu \mathrm{m}^{-2}$ [Fig. 1(a)]. The random antenna arrangement serves to average out the effects of coupling between the individual antennas [16]. The antennas are $200 \mathrm{~nm}$ wide, $100 \mathrm{~nm}$ high, and their length varies between 1400 and $2600 \mathrm{~nm}$. Antennas of these lengths interact strongly with midinfrared light that is polarized along the long antenna axis. 

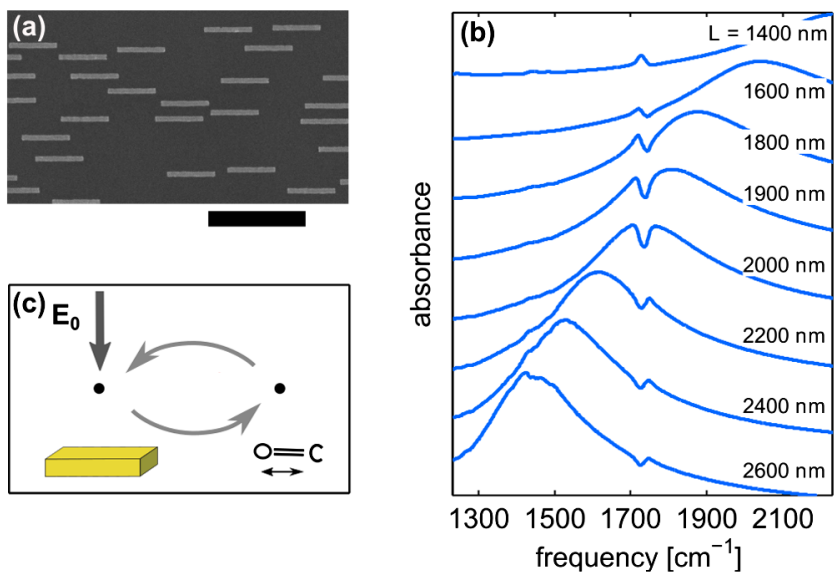

FIG. 1 (color online). (a) SEM image of one of the antenna arrays. The scale bar corresponds to a distance of $4 \mu \mathrm{m}$. (b) Infrared absorbance spectra (light polarized along the long antenna axis) of randomized nanoantenna arrays coated with a $14 \mathrm{~nm}$ layer of PMMA. The spectra are offset vertically for clarity. (c) Schematic representation of the coupled-dipole model described in the text. Both the nanoantenna and the PMMA patch on top are represented as point dipoles.

This is illustrated in Fig. 1(b) where we show the IR transmission spectra of a number of antenna arrays that are covered with a $14 \mathrm{~nm}$ layer of PMMA. These spectra were recorded with a Bruker Vertex 80v FTIR spectrometer coupled to a Hyperion 3000 IR microscope. The longitudinal plasmon resonance has a width of $300-500 \mathrm{~cm}^{-1}$ and the resonance shifts to the red with increasing antenna length [17]. In addition, a small modulation on top of the antenna spectrum is observed around $1730 \mathrm{~cm}^{-1}$, which is due to the interaction with the PMMA carbonyl vibration. The spectra in Fig. 1(b) illustrate that the antennas do not merely amplify the absorption by the PMMA: in that case, one would observe positive absorptive peaks on top of the antenna spectra (i.e., an increased absorption). Instead, the enhancement mechanism leads to complex vibrational line shapes that depend on the detuning between the vibrational transition and the antenna resonance [17]. In the following, we use a simple, coupled-dipole model $[26,27]$ to explain the observed line shapes and their intensities [Fig. 1(c)]. In order to keep the notation simple, we use a scalar description. We treat the antenna and the PMMA patch on top of it as two dipolar point scatterers with respective complex polarizabilities $\alpha_{\text {ant }}(\omega)$ and $\alpha_{\mathrm{vib}}(\omega)$ and locations $r_{\text {ant }}$ and $r_{\text {vib. }}$. We note that both polarizabilities are frequency dependent: $\alpha_{\mathrm{vib}}(\omega)$ due to the vibrational resonance of the CO vibration of PMMA, and $\alpha_{\text {ant }}(\omega)$ due to the plasmonic antenna resonance (tunable by varying the antenna length). The incident electric field $E_{0}(\omega)$ polarizes the antenna, which leads to an enhanced near field that induces a vibrational dipole given by

$$
p_{\mathrm{vib}}(\omega)=\alpha_{\mathrm{vib}}(\omega) G\left(r_{\mathrm{vib}}, r_{\mathrm{ant}}\right) \alpha_{\mathrm{ant}}(\omega) E_{0}(\omega) \text {, }
$$

where $G\left(r_{\mathrm{vib}}, r_{\mathrm{ant}}\right)$ is the Green function for a radiating dipole located at position $r_{\text {ant }}$. In this expression we have neglected the contribution of the incident field itself to the vibrational polarization as its effect is much smaller than that of the local field. The induced vibrational dipole acts back and induces a change in the antenna polarization:

$$
\Delta p_{\text {ant }}(\omega)=\alpha_{\text {ant }}(\omega) G\left(r_{\text {ant }}, r_{\text {vib }}\right) p_{\text {vib }}(\omega) .
$$

Combining these expressions and using the fact that the extinction cross section of the antenna $\sigma_{\text {ant }}(\omega)$ is given by the ratio of the work done on the antenna by the incident field $\frac{1}{2} \omega \operatorname{Im}\left[p_{\text {ant }}(\omega) E_{0}^{*}(\omega)\right]$ to the incident intensity, we arrive at the following expression for the modification of the antenna cross section due to the presence of the PMMA:

$$
\Delta \sigma_{\text {ant }}(\omega)=\frac{2 \pi}{\lambda \epsilon_{0}} \operatorname{Im}\left\{\left[\alpha_{\text {ant }}(\omega) G\right]^{2} \alpha_{\mathrm{vib}}(\omega)\right\}
$$

In this equation $\lambda$ is the free-space wavelength and $\epsilon_{0}$ is the permittivity of free space. We have used the reciprocity relation to equate the two appearing Green functions and simply denoted them by $G$. Despite the approximations made in the above treatment, this equation provides an excellent description of the antenna-enhanced vibrational line shapes, as we have confirmed through finite-difference time domain simulations (see Supplemental Material [28]). Equation (3) shows that the nanoantenna amplifies the original vibrational spectrum by the complex-valued factor $\left(\alpha_{\text {ant }}(\omega) G\right)^{2}$, which represents the square of the field enhancement. Because of the complex nature of the amplification factor, the (enhanced) vibrational signal $\Delta \sigma_{\text {ant }}(\omega)$ typically contains both an absorptive $\left[\operatorname{Im}\left[\alpha_{\mathrm{vib}}(\omega)\right]\right]$ and a dispersive $\left[\operatorname{Re}\left[\alpha_{\mathrm{vib}}(\omega)\right]\right]$ component, as can be seen in Fig. 1(b). It is straightforward to see that there are three circumstances under which purely absorptive line shapes are observed: if the antenna is driven on resonance, far below resonance, or far above resonance. In the first case there is a $\pi / 2$ phase lag between the incident electric field and the antenna polarization: this leads to a negative value of the amplification factor $\left(\alpha_{\text {ant }}(\omega) G\right)^{2}$, so that the vibrational signal is observed as an absorption dip on top of the antenna resonance. Driving the antenna far below resonance or far above resonance results in phase shifts of 0 and $\pi$, respectively, so that in these two circumstances absorptive peaks are observed.

We investigate the nonlinear optical properties of the PMMA-covered arrays using femtosecond IR pump-probe spectroscopy (see the Supplemental Material for a detailed description of the experimental setup [28]). In these measurements we use a femtosecond $(\sim 100 \mathrm{fs}, 150 \mathrm{~nJ})$ infrared pulse centered at $1700 \mathrm{~cm}^{-1}$ to excite a significant fraction of the carbonyl vibrations in the PMMA layer. The pump-induced absorption changes $\left(\Delta \alpha=-\ln T / T_{0}\right)$ are monitored by a delayed probe pulse. In order to perform polarization-resolved measurements, the probe polarization 

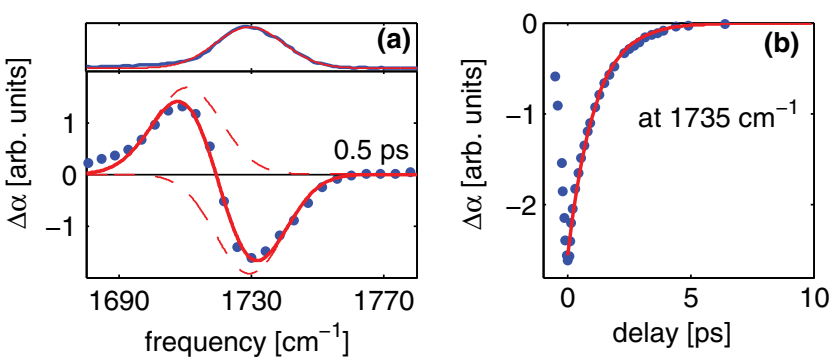

FIG. 2 (color online). (a) Top: Linear infrared spectrum (blue line) of the carbonyl vibration of a $150 \mathrm{~nm}$ film of PMMA on a $\mathrm{CaF}_{2}$ substrate. The red line represents a Gaussian fit. Bottom: Transient spectrum of the same sample (isotropic signal). The solid red line is a fit to a linear combination of two Gaussian bands (dashed lines) whose widths are taken from the linear spectrum. (b) Delay scan of the transient signal at a frequency of $1735 \mathrm{~cm}^{-1}$. The red line is a monoexponential fit.

is set at an angle of 45 deg with respect to the pump polarization. Using polarizers behind the sample we then select the probe component that is polarized either parallel or perpendicular to the pump polarization. Scattered pump light is suppressed by rapidly modulating the pump-probe delay by half an optical period [29].

As a reference measurement the pump-probe spectrum of a thick layer of PMMA $(\sim 150 \mathrm{~nm})$ deposited on a $\mathrm{CaF}_{2}$ substrate is shown in Fig. 2. We observe the characteristic transient spectrum of an anharmonic vibration. At the fundamental frequency $\left(1730 \mathrm{~cm}^{-1}\right)$ there is a decreased absorption due to the depletion of the ground state and stimulated emission from the vibrationally excited state. At lower frequencies $\left(\sim 1710 \mathrm{~cm}^{-1}\right)$ an induced absorption is observed due to absorption by the excited state. A fit of the transient spectrum to a linear combination of two Gaussian bands reveals an anharmonicity of $19 \mathrm{~cm}^{-1}$. The ratio of the $0-1$ and $1-2$ cross sections is 1.8 , which is very close to the value of 2 expected for a purely harmonic vibration. Figure 2(b) shows a delay scan for a frequency corresponding to the peak of the bleach $\left(\sim 1735 \mathrm{~cm}^{-1}\right)$. We observe a monoexponential decay with a time constant of $1.1 \mathrm{ps,}$ which we attribute to the vibrational relaxation of the carbonyl vibration.

Having characterized the transient vibrational response of PMMA itself, we move on to study the effect of coupling with the nanoantennas. For this purpose we work with a $5 \mathrm{~nm}$ film of PMMA (see Supplemental Material for the determination of the thickness [28]), which gives no appreciable pump-probe signal in the absence of antennas: the smallest signal we could detect $\left(\Delta \alpha=2 \times 10^{-5}\right)$ was for a $10 \mathrm{~nm}$ thick film, which is shown in Fig. 3(a). Next we consider the situation of a $5 \mathrm{~nm}$ film deposited on an array of nanoantennas $(L=2000 \mathrm{~nm})$ that are tuned to resonance with the carbonyl vibration. Figure 3(b) shows the pump-probe signal for a measurement in which the pump pulse is polarized parallel to the long axis of the antennas
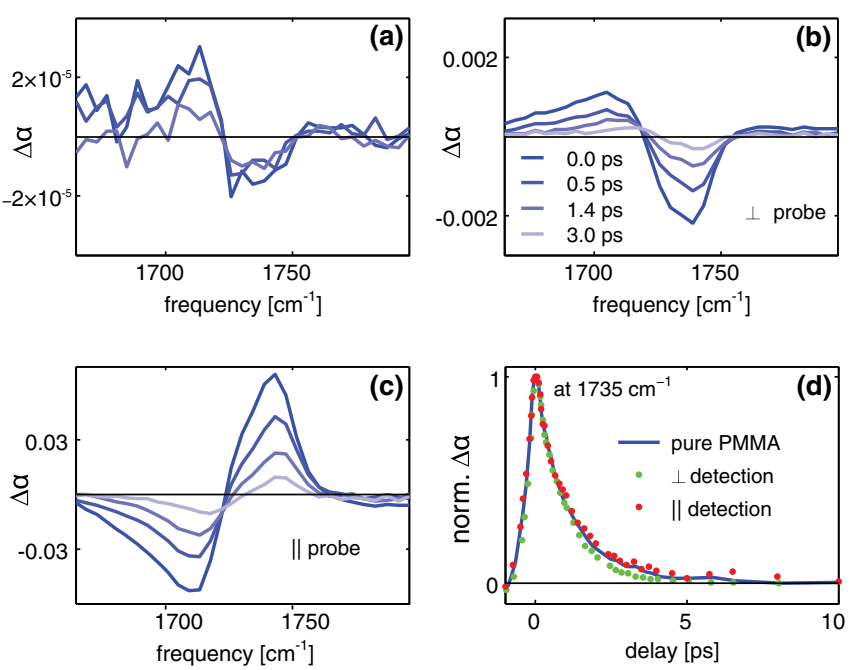

FIG. 3 (color online). (a) Transient spectrum of a $10 \mathrm{~nm}$ layer of PMMA on $\mathrm{CaF}_{2}$ (isotropic signal). (b) Transient signal for a $5 \mathrm{~nm}$ PMMA film deposited on top of nanoantennas. The pump polarization is along the long axis of the antenna while the probe polarization is along the short axis. (c) Transient signal for the same sample as in (b) with the pump and probe polarizations set parallel to the long axis of the antennas. (d) Normalized delay traces at $1735 \mathrm{~cm}^{-1}$ for the reference measurement on a $150 \mathrm{~nm}$ layer of PMMA (blue line) and for a $5 \mathrm{~nm}$ layer of PMMA on a nanoantenna array $(L=2000 \mathrm{~nm})$ with a perpendicular probe (red dots) and a parallel probe (green dots).

and the probe pulse is polarized along the short antenna axis. Remarkably, even though the (perpendicularly polarized) probe is not resonant with the antennas, we nevertheless observe an increase in the nonlinear signal by a factor of 100. Next we polarize the probe along the long axis of the nanoantennas [Fig. 3(c)]. We observe a further increase of the nonlinear signal to a value as high as $\Delta \alpha=0.055$. In addition, we see that the sign of the nonlinear signal is inverted compared to the reference signals in Figs. 2(a) and 3(a). This inversion can be understood from the linear spectrum of the $2000 \mathrm{~nm}$ antennas in Fig. 1(b). As was explained above, in this case the coupling to the nanoantennas is such that the presence of the carbonyl vibration leads to a decrease of the absorption. As a consequence, the depletion of the ground state of this vibration by a pump pulse leads to an increased absorption while the coupling of the antennas with the newly accessible $1 \rightarrow 2$ transition leads to a decreased absorption. We note that when the antennas are probed along their short axis, we also observe an enhancement of the nonlinear signal [Fig. 3(b)]. In this case the nonlinear signal has the regular sign: a decreased absorption at the fundamental frequency and an increased absorption at the excited-state frequency. This nonlinear signal originates from the coupling of the excited vibrations (and the depleted ground state) with the transverse resonance of the nanoantennas, which lies at much higher frequencies in 
the visible spectral region [30]. The normal sign follows from the fact that the vibrations drive this transverse antenna mode far below its resonance frequency, which, according to Eq. (3), leads to a positive (and real-valued) amplification factor in the linear spectrum and therefore also in the nonlinear spectrum.

It should be noted that the nonlinear signal in Fig. 3(c) originates from only a small fraction of the $\mathrm{CO}$ vibrations on the substrate (i.e., those vibrations located close to the antenna surface). The nonlinear signal enhancement is, therefore, much larger than the ratio of the absorption changes in Figs. 3(a) and 3(c). The question arises of how large the enhancement actually is. The nonlinear signal is described by an equation identical to Eq. (3) except for the replacement of $\alpha_{\mathrm{vib}}(\omega)$ by the pump-induced change $\Delta \alpha_{\mathrm{vib}}(\omega)$, which is proportional to the number of excited vibrations and therefore to the local field intensity. Since $\alpha_{\text {ant }}(\omega) G$ represents the field enhancement, the nonlinear signal is proportional to the fourth power of the field enhancement. Using this result in combination with finitedifference time domain simulations, we find that more than $90 \%$ of the nonlinear signal originates from CO vibrations that are within $40 \mathrm{~nm}$ of the antenna surface. This means that the nonlinear signal in Fig. 3(c) originates from $10 \%$ of the $\mathrm{CO}$ vibrations covering the antenna array (the nanoantennas cover $7 \%$ of the surface), from which a net enhancement factor of $6 \times 10^{4}$ for the nonlinear spectrum in Fig. 3(c) can be determined (see Supplemental Material for details [28]).

Next, we turn to the time dependence of the enhanced vibrational signal. An important point is whether the temporal decay of the signal tracks the excited state population of the carbonyl vibration or whether it is affected nontrivially by the coupling with the nanoantenna. To investigate this issue we compare the delay traces of the enhanced signals (i.e., for parallel and perpendicular probing) with the delay trace of the unenhanced reference measurement [Fig. 3(d)]. The strong resemblance of the three signals demonstrates that the enhanced signals indeed accurately reflect the intrinsic vibrational dynamics of the molecular system (and therefore provide information about the molecular system itself).

The high signal strength and excellent signal-tonoise ratio of the antenna-enhanced pump-probe spectra [Fig. 3(c)] allow us to move beyond one-color pump-probe spectroscopy and explore the capabilities of antenna enhancement in 2DIR spectroscopy. We employ a pumping technique in which a Mach-Zehnder interferometer is used to generate a pair of collinear pump pulses [31]. Transient spectra are recorded as a function of the delay between the two pump pulses, and Fourier transformation of this time variable yields the pump-frequency axis in the twodimensional spectrum. Figure 4 shows examples of 2DIR spectra for two different antenna fields $(L=1900 \mathrm{~nm}$ and $L=2200 \mathrm{~nm}$ ) coated with $5 \mathrm{~nm}$ of PMMA. The
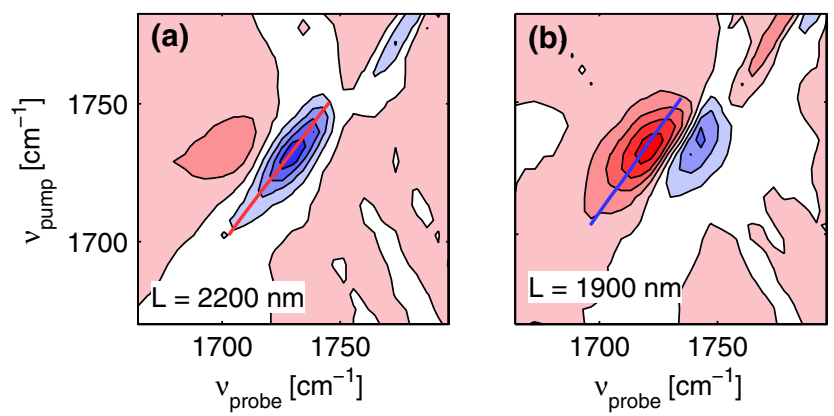

FIG. 4 (color online). 2DIR spectra of $5 \mathrm{~nm}$ PMMA on nanoantennas of (a) $2200 \mathrm{~nm}$ and (b) $1900 \mathrm{~nm}$. The thin solid lines represent equidistantly spaced contours. Positive absorption changes are shown in red and negative absorption changes in blue. The spectra correspond to a pump-probe delay of $0.5 \mathrm{ps}$. The thick solid lines represent the central lines and are calculated by fitting the locations of the local maxima of the $2 \mathrm{D}$ spectra to a straight line.

pronounced diagonal elongation in these spectra indicates that the carbonyl band of PMMA is in fact composed of an inhomogeneous distribution of $\mathrm{CO}$ oscillators that can be excited independently of each other: the band shows strong inhomogeneous broadening. In passing, we note that a homogeneously broadened band would show a nearly vertical central line. A measure for the degree of inhomogeneous broadening is provided by the tilt of the central line, which is drawn in the 2D spectra. The two spectra in Fig. 4 exhibit different two-dimensional line shapes reflecting the difference in the corresponding linear line shapes. Nevertheless, the central line slope is identical for both spectra $(1.2 \pm 0.1)$, indicating that this parameter can be extracted quite robustly from the antenna-enhanced 2D spectra. In the future, it will be interesting to perform these measurements as a function of the pump-probe delay, which will provide information about the time scale on which spectral fluctuations occur.

An obvious question that arises after these proof-ofprinciple experiments is how far we are removed from performing nonlinear vibrational spectroscopy on molecular monolayers, such as supported lipid membranes. From the density and molecular weight of PMMA, we estimate that a $5 \mathrm{~nm}$ layer of PMMA contains $\sim 35 \mathrm{CO}$ groups $/ \mathrm{nm}^{2}$. For a lipid bilayer this number is $\sim 6 \mathrm{CO}$ groups $/ \mathrm{nm}^{2}$, as can be deduced from the typical surface area of $0.7 \mathrm{~nm}^{2}$ per lipid molecule [32]. This comparison shows that we are already within an order of magnitude of the required sensitivity. Given that the nonlinear signal in our experiments scales with the fourth power of the field enhancement, an obvious way to increase the sensitivity would be to use nanostructures which provide higher field enhancements. Examples of structures that could provide exceptionally high field enhancements are optimally coupled arrays of nanoantennas [30], fractal-shaped antennas [19], and perfect-absorber metamaterials [33]. In conclusion, 
we have demonstrated that metallic nanoantennas can be used to amplify nonlinear vibrational signals by more than 4 orders of magnitude. This new technique will allow the study of molecular structures and dynamics of nanoscopic volumes including molecular monolayers.

This work is part of the research program of the "Stichting voor Fundamenteel Onderzoek der Materie" (FOM), which is financially supported by the "Nederlandse organisatie voor Wetenschappelijk Onderzoek" (NWO). The authors thank Huib Bakker and Kobus Kuipers for useful discussions and for critically reading the manuscript, and Jan Helbing for sharing his designs of the Mach-Zehnder interferometer and wobbling Brewster plate.

*rezus@amolf.nl

[1] L. Szyc, M. Yang, E. T. J. Nibbering, and T. Elsaesser, Angew. Chem., Int. Ed. Engl. 49, 3598 (2010).

[2] K. J. Tielrooij, N. Garcia-Araez, M. Bonn, and H. J. Bakker, Science 328, 1006 (2010).

[3] J. Manor, P. Mukherjee, Y.-S. Lin, H. Leonov, J. L. Skinner, M. T. Zanni, and I. T. Arkin, Structure 17, 247 (2009).

[4] P. Hamm, J. Helbing, and J. Bredenbeck, Annu. Rev. Phys. Chem. 59, 291 (2008).

[5] A.W. Smith, J. Lessing, Z. Ganim, C. S. Peng, A. Tokmakoff, S. Roy, T. L.C. Jansen, and J. Knoester, J. Phys. Chem. B 114, 10913 (2010).

[6] J. R. Zheng, K. Kwak, J. Asbury, X. Chen, I. R. Piletic, and M. D. Fayer, Science 309, 1338 (2005).

[7] A. Remorino, I. V. Korendovych, Y. Wu, W. F. DeGrado, and R. M. Hochstrasser, Science 332, 1206 (2011).

[8] S. Woutersen and P. Hamm, J. Phys. Chem. B 104, 11316 (2000).

[9] Y. L. A. Rezus and H. J. Bakker, Phys. Rev. Lett. 99, 148301 (2007).

[10] J. C. Love, L. A. Estroff, J. K. Kriebel, R. G. Nuzzo, and G. M. Whitesides, Chem. Rev. 105, 1103 (2005).

[11] M. A. Cohen Stuart, W. T. S. Huck, J. Genzer, M. Mueller, C. Ober, M. Stamm, G. B. Sukhorukov, I. Szleifer, V. V. Tsukruk, M. Urban, F. Winnik, S. Zauscher, I. Luzinov, and S. Minko, Nat. Mater. 9, 101 (2010).

[12] D. E. Rosenfeld, Z. Gengeliczki, B. J. Smith, T. D. P. Stack, and M. D. Fayer, Science 334, 634 (2011).

[13] J. P. Kraack, D. Lotti, and P. Hamm, J. Phys. Chem. Lett. 5, 2325 (2014).

[14] P. Biagioni, J.-S. Huang, and B. Hecht, Rep. Prog. Phys. 75, 024402 (2012).
[15] P. Bharadwaj, B. Deutsch, and L. Novotny, Adv. Opt. Photonics 1, 438 (2009).

[16] R. Adato, A. A. Yanik, J. J. Amsden, D. L. Kaplan, F. G. Omenetto, M. K. Hong, S. Erramilli, and H. Altug, Proc. Natl. Acad. Sci. U.S.A. 106, 19227 (2009).

[17] F. Neubrech, A. Pucci, T. W. Cornelius, S. Karim, A. García-Etxarri, and J. Aizpurua, Phys. Rev. Lett. 101, 157403 (2008).

[18] L. V. Brown, K. Zhao, N. King, H. Sobhani, P. Nordlander, and N. J. Halas, J. Am. Chem. Soc. 135, 3688 (2013).

[19] H. Aouani, H. Sipova, M. Rahmani, M. Navarro-Cia, K. Hegnerova, J. Homola, M. Hong, and S. A. Maier, ACS Nano 7, 669 (2013).

[20] D. Dregely, F. Neubrech, H. Duan, R. Vogelgesang, and H. Giessen, Nat. Commun. 4, 2237 (2013).

[21] A. Bouhelier, M. Beversluis, A. Hartschuh, and L. Novotny, Phys. Rev. Lett. 90, 013903 (2003).

[22] M. Lippitz, M. A. van Dijk, and M. Orrit, Nano Lett. 5, 799 (2005).

[23] M. Danckwerts and L. Novotny, Phys. Rev. Lett. 98, 026104 (2007).

[24] Y. Zhang, F. Wen, Y.-R. Zhen, P. Nordlander, and N. J. Halas, Proc. Natl. Acad. Sci. U.S.A. 110, 9215 (2013).

[25] P. M. Donaldson and P. Hamm, Angew. Chem., Int. Ed. Engl. 52, 634 (2013).

[26] P. Alonso-Gonzalez, P. Albella, M. Schnell, J. Chen, F. Huth, A. Garcia-Etxarri, F. Casanova, F. Golmar, L. Arzubiaga, L. E. Hueso, J. Aizpurua, and R. Hillenbrand, Nat. Commun. 3, 684 (2012).

[27] N. T. Fofang, N. K. Grady, Z. Fan, A. O. Govorov, and N. J. Halas, Nano Lett. 11, 1556 (2011).

[28] See Supplemental Material at http://link.aps.org/ supplemental/10.1103/PhysRevLett.114.233004 for the comparison of the coupled point-dipole model with the FDTD simulations; details about the thickness determination of the PMMA layers; details about the experimental setup; and details about the calculation of the enhancement factor of the nonlinear signal.

[29] R. Bloem, S. Garrett-Roe, H. Strzalka, P. Hamm, and P. Donaldson, Opt. Express 18, 27067 (2010).

[30] C. D'Andrea, J. Bochterle, A. Toma, C. Huck, F. Neubrech, E. Messina, B. Fazio, O. M. Marago, E. Di Fabrizio, M. L. de la Chapelle, P. G. Gucciardi, and A. Pucci, ACS Nano 7, 3522 (2013).

[31] J. Helbing and P. Hamm, J. Opt. Soc. Am. B 28, 171 (2011).

[32] N. Kucerka, M.-P. Nieh, and J. Katsaras, Biochim. Biophys. Acta 1808, 2761 (2011).

[33] K. Chen, R. Adato, and H. Altug, ACS Nano 6, 7998 (2012). 